\title{
VII.
}

\section{Sur une prétendue faute de raisonnement que Descartes aurait commise.}

Par

J. Chazottes, professeur à Guérêt (Creuse).

Dans une lettre de Descartes, en français, qui porte le $\mathrm{n}^{0} \mathrm{XIV}$ et la date du 13 novembre 1629 dans l'édition des CEuvres de ce philosophe publiée par MM. Charles Adam et Paul Tannery, sous les auspices du Ministère de l'Instruction publique, se trouve contenu un fragment latin relatif à la loi mathématique de la chute des graves dans le vide qui, d'après M. P. Tannery, serait d'une rédaction bien antérieure et remonterait à l'époque du premier séjour de Descartes en Hollande (de 1617 à juillet 1619). Voici ce fragment:

¿Quod in vacuo semel incoepit moveri, semper et aequali celeritate movetur. Supponas ergo pondus in $A$ (Fig. 1) existens impelli a sua gravitate versus $C$. Dico statim atque coepit moveri, si desereret illum (sic) ipsius gravitas, nihilominus pergeret in eodem motu donec perveniret ad $C$; sed tunc non tardius nec celerius descenderet ab. $A$ ad $B$

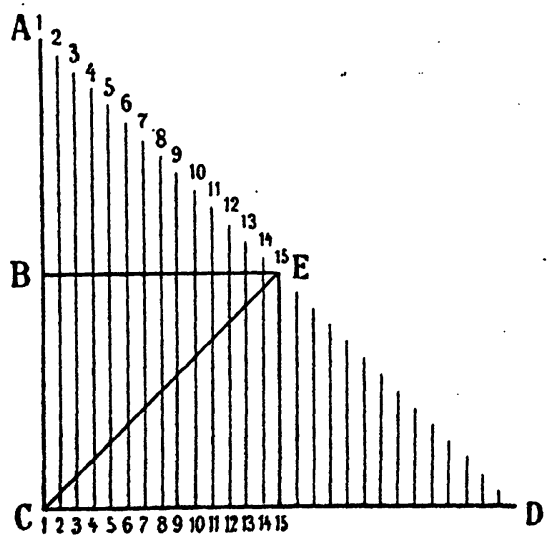

Fig. 1. quam a $B$ ad $C$. Quia vero non ita fit, sed adest illi gravitas quae premit illum (sic) deorsum et addit singulis momentis novas vires ad descendendum, hinc fit ut multo celerius absolvat spatium $B C$ quam $A B$, quia in eo percurrendo retinet omnem impetum quo movetur per spatium 
$A B$ et insuper novus ei accrescit propter gravitatem quae de novo urget singulis momentis. Qua autem proportione augeatur ista celeritas, demonstratur in triangulo $A B C^{\prime} D E$ : nempe prima linea denotat vim celeritatis impressam $1^{0}$ momento, $2^{0}$ linea vim impressam $2^{0}$ momento, $3^{n}$ vim $3^{0}$ inditam, et sic consequenter. Unde fit triangulus $A C D$ qui repraesentat augmentum celeritatis motus in descensu ponderis ab $A$ usque ad $C$, et $A B E$ qui repraesentat augmentum celeritatis in priori media parte spatii quod pondus percurrit, et trapezium $B C D E$ quod repraesentat augmentum celeritatis in posteriori media parte spatii quod pondus percurrit, nempe $B C$. Et cum trapezium $B C D E$ fit triplo majus triangulo $A B E$, ut patet, inde sequitur pondus triplo celerius descensurum a $B$ ad $C$ quam ab $A$ ad $B$; idest, si tribus momentis descendit ab $A$ ad $B$, unico momento descendet a $B$ ad $C$; idest quattuor momentis duplo plus itineris conficiet quam tribus, et per consequens 12 momentis duplo plus quam 9 , et 16 momentis quadruplo plus quam 9 , et sic consequenter."

Au sujet de ce passage, M. Tannery fait la remarque suivante: "Dans cette recherche a priori (de la loi mathématique de la chute des graves dans le vide), Descartes procède comme paraît l'avoir fait aussi Galilée, en partant du principe de la conservation du mouvement antérieurement acquis, et en employant un procédé tout à fait analogue à celui de la méthode des indivisibles (ainsi bien avant Cavalieri). Mais il commet une faute de raisonnement singulière. Sur sa figure, la coordonnée $A B C$ devrait représenter les temps, tandis qu'il s'en sert également pour représenter les espaces parcourus. La marche; très ingénieuse au reste, qu'il suit, l'empêche d'apercevoir immédiatement les cọntradictions auxquelles cette confusion sur la figure aurait dû le conduire; il aboutit donc à une relation essentiellement différente de celle de Galilée, puisqu' elle reviendrait à considérer l'espace parcouru comme proportionnel, non pas au carré du temps, mais à une puissance du temps dont l' exposant est le rapport de $\log .2$ à log. $\frac{4}{3}$, c'est-à-dire environ 2,4 .

Ayant depuis longtemps rejeté l'hypothèse de la possibilité du : vide, Descartes ne revint jamais sérieusement sur ce tentamen, 
et par suite ne reconnut pas son erreur. Il semble même avoir cru de bonne foi que la loi de Galilée ne différait pas de celle qu'il avait lui-même donnée à Beeckmann dès 1619."

Descartes a-t-il, réellement, commis la „faute de raisonnement singulière" qu'on lui reproche? Cela ne laisserait pas d'être déjà quelque peu surprenant de la part de l'auteur du Discours de la méthode; mais, ce qui aurait encore bien plus lieu de nous surprendre c'est que, l'ayant commise, il ait pu croire "de bonne foi que la loi de Galilée ne différait pas de celle qu'il avait lui-même donnée“. Il faudrait admettre, en effet, ou bien qu'il n'a pas bien compris Galilée, ou bien qu'il ne s'est pas bien compris lui-même. Nous ne voyons pas bien, d'autre part, comment le fait que Descartes avait depuis longtemps rejeté l'hypothèse de la possibilité du vide aurait pu l'empêcher de revenir "sur ce tentamen" et de reconnaître „son erreur". L'étude de la manière dont se produirait la chute d'un corps dans le vide n'est, comme celle de la manière dont elle se produirait, à supposer que le corps fût, dès l'instant même où il est mis en mouvement par l'action de la pesanteur, soustrait à cette action, qu'une application de la $2^{\mathrm{e}}$ règle de la méthode, telle qu'elle sera formulée plus tard dans le Discours: "Diviser chacune des difficultés que j'examinerais en autant de parcelles qu'il se pourrait, et qu'il serait requis pour les mieux résoudre." Que le vide puisse ou non exister, en fait, cette étude n'en conserve pas moins son utilité pour permettre de déterminer la manière dont la chute se produit, dans les conditions plus complexes où el lese présente dans la réalité. Si donc Descartes avait réellement commis une erreur, on ne voit pas que rien ait pu l'empêcher de la reconnaître; et il n'est guère douteux que la publication ultérieure (1632) de la formule donnée par Galilée eût dû tout naturellement lui en fournir l'occasion. Mais l'erreur ne serait-clle pas plutôt tout entière du fait de $\mathrm{M}$. Tannery et ne serait-elle pas due simplement à ce que le commentateur n'a pas sérieusement essayé de se placei au point de vue de l'auteur dont il discutait la doctrine?

„Sur la figure de Descartes, dit M. Tannery, la coordonnée $A B C$ devrait représenter les temps, tandis qu'il s'en sert également pour représenter les espaces parcourus." Descartes se sert, en effet, de 
la coordonnée $A B C$ pour représenter les espaces parcourus, mais par le poids $A$ supposé d'un mouvement uniforme, auquel cas les espaces sont proportionnels aux temps, ce qui fait que la même coordonnée peut représenter indifféremment les uns et les autres. Quant aux espaces parcourus dans le mouvement du même poids $A$, tel qu'il se produirait en fait dans le vide (que le vide existe ou non réellement peu importe), sous l'action de la pesanteur, ils sont représentés par les surfaces $A B E, A B C D E$ etc. etc., des triangles semblables successivement construits sur la coordonnnée $A B C$.

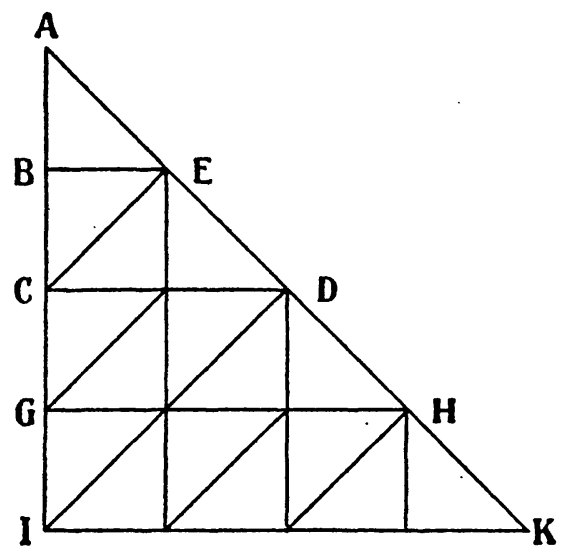

Fig. 2.

Il suffit de jeter un coup d'œil sur la figure 2 pour s'assurer -que ces espaces sont, à la fin de la première unité de temps, 1 ; - à la fin de la seconde, 4 ; à la fin de la $3^{\ominus}$, 9: à la fin do la $4^{\ominus}, 16$ etc. etc.; c'est-à-dire qu'ils sont proportionnels aux carrés des temps employés à les parcourir. Ce n'est pas autre chose, au fond, que dit Descartes: il s'exprime seulement en termes différents.

Descartes suppose un poids $A$ qui tombe vers $C$ (Fig. 3) dans des conditions telles que, dès l'instant même où il est mis en mouvement par l'action de la pesanteur, il soit soustrait à cette A T action. Ce poids franchira l'espace $A C$ dans un temps qui sera juste le double de celui qu'il lui aurait fallu pour franchir la moitié $A B$ de ce même espace. Si donc il a mis une unité de temps pour aller de $A$ en $B$, il en mettra 2 pour achever d'arriver en $C$. Ceci posé, Descartes se demande combien il faudrait de temps à ce même poids $\dot{A}$ pour franchir le même espace $A C$ dans les $G$ conditions réelles de sa chute, c telle qu'elle se produirait dans le vide (à supposer que le Fig.3. vide existât): il trouve que, par suite de l'accélération due à la pesanteur, il lui faudrait trois fois moins de temps pour passer de $B$ en $C$ qu'il ne lui en aurait fallu pour passer de $A$ en $B$; c'est-à-dire que, supposè qu'il lui ait fallu 3 unités de temps pour passer de $A$ en $B$, il ne lui en faudrait plus qu'une pour 
Sur une prètendue faute de raisonnement que Descartes aurait commise. 175

passer de $B$ en $C$. En d'autres termes, le poids franchirait l'intervalle $A C$ dans les $G \frac{4}{3}$ du temps qu'il lui aurait fallu pour en franchir la moitié $A B$, ou, ce qui revient au même, il parcourrait $4 A B$ $\left(=A C_{2}\right)$ dans le temps qu'il aurait mis à en parcourir $2\left(=A C_{1}\right)$. Raisonnant sur $A C_{2}$ comme sur $A C_{1}$, on trouve que le poids $A$ franchirait l'intervalle $A C_{2}$ dans les $\frac{4}{3}$ du temps qu'il lui aurait fallu pour en franchir la moitiée $A C_{1}(=2 A B)$, c'est-à-dire dans les $G \frac{4}{3} \times \frac{4}{3}=\frac{16}{9} \mathrm{du}$ temps qu'il lui aurait fallu pour parcourir $A B\left(=\frac{A \biguplus_{\mathrm{i}}}{4}\right)$, ou, ce qui revient au même, il parcourrait $16 \mathrm{AB}$ dans le temps qu'il aurait mis à en parcourir 4, c'est-à dire dans 4 unités de temps. Les espaces parcourus seraient donc proportionnels aux carrés des temps employés à les $G$ parcourir.

On s'explique, après cela, que Descartes ait pu croire „de bonne foi que la loi de Galilée ne différait pas de celle qu'il avait lui-même donnée à Beeckmann dès 1619.“ 